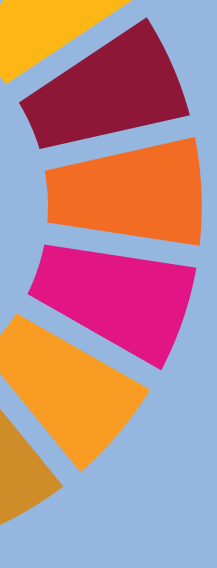

\title{
EL IMPACTO DE LA
}

INHELICENCIA ARTIFICIAL

\author{
EN LA SOCIEDAD Y \\ SU APLICACIÓN EN EL \\ SECTOR FINANCIERO
}

\author{
THE IMPACT OF \\ ARTIFICIAL INTHLLIGENCE \\ ON SOCIETY AND ITS \\ APPLICATION IN THE \\ FINANCIAL SECTOR \\ María Asunción Gilsanz Muñoz \\ Mediterraneo Vida \\ magilsanz@medvida.es
}

\section{RESUMEN}

Como impactarán las nuevas tecnologías en la sociedad en los próximos añoso como podrála Inteligencia Artificial ayudar a sectores como la economía o el medio ambiente es una pregunta que todos nos estamos haciendo.

La Inteligencia Artificial ya es parte de nuestro presente y será parte clave de nuestro futuro, pero ¿qué ha cambiado para que esto haya sido posible? La respuesta está en el avance que la tecnología ha tenido en los últimos años debido al incremento exponencial que han tenido tres factores clave, la potencia de procesamiento, el aumento de la velocidad en las comunicaciones y el abaratamiento del almacenamiento de datos, que han facilitado la innovación tecnológica.

Esta facilidad para la innovación tecnológica ha propinado que hoy en día el mercado está lleno de pequeñas empresas o startups que están aprovechando esta tecnología para desarrollar nuevos productos y soluciones que mejoren la vida de la sociedad y de las personas.

Palabras clave: Inteligencia Artificial, Tecnología, Impacto, Sector financiero, Ética 


\section{SUMMARY}

How new technologies will impact society in the coming years or how Artificial Intelligence can help sectors such as the economy or the environment is a question we all ask ourselves.

Artificial intelligence is already part of our present and will be a key part of our future, but what has changed to make this possible? The answer lies in the advancement that technology has had in recent years due to the exponential increase in three key factors, processing power, the increase in speed in the communications and the cheapening of data storage, which have facilitated technological innovation.

This facility for technological innovation has made the market today full of small companies or startups that are taking advantage of this technology to develop new products and solutions that improve the lives of society and people.

Keywords: Artificial intelligence, Technology, Impact, Financial sector, Ethics

Maria Asunción Gilsanz. Licenciada en Ciencias Matemáticas y master en Finanzas Cuantitativas. Especialista en sistemas de información, nuevas tecnologías e implantación de soluciones de robotización e Inteligencia Artificial en empresas del sector financiero. Ha liderado grandes proyectos de consultoría tecnológica a nivel internacional y departamentos de tecnología e innovación en bancos como Santander o Sabadell. Le apasiona impulsar la transformación digital a través de la innovación y el uso ético de las nuevas tecnologías.

\section{1. ¿CÓMO VA A PODER AYUDAR LA INTELIGENCIA ARTIFICIAL A LA SOCIEDAD Y CÓMO SERÁ NUESTRO FUTURO?}

El uso de la Inteligencia Artificial hoy en día ya es clave en campos como la robótica. Estos sistemas utilizan la Inteligencia Artificial para realizar tareas sencillas y de poco valor, como por ejemplo la carga de datos en los sistemas de gestión de una empresa, o para realizar tareas repetitivas que requieren un esfuerzo físico, como por ejemplo en el ensamblaje de piezas en una cadena de suministro. Y si hablamos de los sistemas de aprendizaje automático y de redes neuronales, su uso está siendo cada vez más extendido en aplicaciones de reconocimiento de voz y análisis de sentimiento, en sistemas de visión artificial y de análisis predictivo. Estos sistemas están dando lugar a aplicaciones como chatbots que interactúan con nosotros por medio de la voz, drones que toman imágenes de lugares poco accesibles, aplicaciones capaces de detectar patrones de fraude, $y$ aplicaciones que ayudan a la prevención del cáncer o más recientemente la predicción de evolución del COVID-19.

La tecnología digital produce siempre beneficios sociales y emocionales para el individuo, y por ello su uso se ha expandido rápidamente a todos los sectores de la sociedad. Su impacto ha sido más visible en algunos ámbitos como la domótica, el transporte o el sector salud, pero sabemos que se extenderá rápidamente a otros sectores como la agricultura, el sector financiero y la educación.

Si ahondamos un poco más en cómo la Inteligencia Artificial impactará en estos tres últimos sectores nos damos cuenta de cómo esta tecnología será clave por ejemplo en la agricultura. En este sector, el futuro estará marcado por el uso de aplicaciones como los drones y el GPS para el escaneo de suelo, el uso de 
la robótica para la siembra y recogida de la cosecha y el uso del internet de las cosas en los sensores que monitorearán el clima y el suelo en tiempo real, para ajustar el riego o la cantidad de fertilizante a utilizar. El uso de la Inteligencia Artificial en este ámbito creará una "agricultura digital" (Trendov, Varas, \& Zeng, 2019) basada en sistemas sumamente productivos, prospectivos y adaptables a los cambios, pudiendo mejorar los cultivos de las zonas más desfavorecidas del planeta optimizando la producción y los recursos para hacerlos más sostenibles.

Continuando con el uso de la Inteligencia Artificial en los colectivos más desfavorecidos, dentro del ámbito socioeconómico se está trabajando desde hace años en la inclusión financiera de personas vulnerables en países emergentes. En esta línea se podrían desarrollar algoritmos de Inteligencia Artificial que utilicen los datos demográficos históricos para identificar segmentos de la población vulnerables, pero con capacidad de emprender pequeños negocios. Con esta información, un banco que hubiera identificado un segmento del tejido productivo (por ejemplo, mujeres con hijos con negocios en el sector textil en determinada zona geográfica) para complementar la información del algoritmo y desarrollar un producto de microcréditos dirigido a este colectivo. Además, si quisiera minimizar aún más su riesgo de crédito podría ofrecer no solo financiación, sino también asesoramiento, formación financiera y seguimiento a través de una plataforma de educación financiera que ayudase a estas mujeres a emprender su nuevo negocio de manera exitosa.

Como se muestra en este ejemplo la educación es clave para el futuro de nuestra sociedad y en este ámbito la Inteligencia Artificial deberá jugar un papel relevante dentro de la nueva estrategia formativa, desarrollando formación accesible para todos, apoyando las capacidades de los profesores o fomentando la innovación entre los alumnos. De la misma forma que la digitalización está teniendo impacto en el trabajo lo está teniendo en la educación, ya que, como resultado de la digitalización, se acentuará la necesidad de una educación más especializada y capacitación de alta calidad para los docentes. Según el informe sobre el impacto de la Inteligencia Artificial en la educación (Cornieles, 2019) "el Gobierno deberá promover la formación en habilidades digitales en todas las fases del sistema educativo, invertir en la formación continua de los docentes ante los nuevos requerimientos tecnológicos y profesionales del mercado y adaptar los contenidos educativos al ámbito digital y a las habilidades emprendedoras. La Inteligencia Artificial debe jugar un papel central en dicha estrategia formativa."

Pero más allá de lo que sabemos que tendrá que ocurrir de manera natural debido a la digitalización, el valor que aportará la Inteligencia Artificial en el ámbito de la educación estará en que la educación podrá acceder a colectivos "no estándar" a donde antes le era muy difícil llegar, como por ejemplo como comentábamos anteriormente los colectivos vulnerables, ofreciendo una oportunidad de desarrollo intelectual adaptado a las necesidades personales de cada individuo. Un ejemplo de cómo la Inteligencia Artificial podría impactar en el desarrollo intelectual es el método Sancal (Roca, 2019) que utiliza la música para crear algoritmos de Inteligencia Artificial que determinen el mejor diagnóstico y programa para niños con problemas de autismo o para personas mayores con problemas de deterioro neuronal y que también podría ser aplicado a futuro en nuestras aulas para el apoyar a los más pequeños en las primeras fases de su desarrollo cognitivo. 


\section{CÓMO LA INTELIGENCIA ARTIFICIAL Y LA DIGITALIZACIÓN IMPACTARÁ AL SECTOR FINANCIERO}

También sectores económicos como el financiero o el asegurador están usando la Inteligencia Artificial para mejorar sus modelos de negocio, sus modelos de atención al cliente y reducir costes operativos.

Cada día más clientes utilizan dispositivos digitales para sus operaciones habituales, para el asesoramiento en inversiones o para conocer su posición global unificando la información de todos sus productos o hacer una predicción del gasto y ahorro futuro mediante soluciones de planificación financiera. De hecho, el entorno digital ha superado ya a la sucursal como principal canal para determinados segmentos claves como la generación Y (los millennials) y el móvil es el principal canal para realizar gestiones bancarias digitales (59\%), seguido del ordenador (32\%) y por último la tablet (7\%) (Sánchez, 2020).

A todos nos gusta que nos ofrezcan un buen servicio y más aún cuando se trata de nuestro dinero. Y es por eso, por lo que ya se están desarrollando aplicaciones que ofrecen a los clientes asesoramiento financiero personalizado para sus inversiones, adaptándolas a sus necesidades reales y a su perfil de riesgo. Estas aplicaciones utilizan algoritmos de Inteligencia Artificial que analizan los datos del mercado junto con los datos de comportamiento del cliente con el fin realizar la mejor inversión según el perfil del cliente y al mejor precio. Un ejemplo de este tipo de aplicaciones es el robo advisors de Unicaja (Porras, 2020).

También encontramos aplicaciones de Inteligencia Artificial que agrupan saldos y movimientos de diferentes bancos y que buscan ayudarnos a controlar mejor nuestros gastos, a darnos pautas de ahorro y que analizan nuestros movimientos para alertarnos de cualquier anomalía, como por ejemplo un recibo de una factura de luz duplicado. Fintonic es una de las aplicaciones más extendidas que usa la Inteligencia Artificial con dichos fines y que está extendiendo su modelo de negocio ofreciendo servicios para el cliente como préstamos o seguros basados en su comportamiento.

Sabemos que el futuro será la interacción por voz, dejaremos de escribirnos mensajes y empezaremos a interactuar con nuestras aplicaciones y a operar con nuestros bancos a través de un bot que hablará con nosotros de manera natural y sin errores. Podremos hablar a nuestra asistente por voz y encargarle la cena, y dejar que ella nos sugiera los mejores restaurantes según nuestros gustos, nuestra asistente también podrá hacer una transacción bancaria si se lo pedimos, o realizar una compra online. Esto poco a poco mejorará nuestra experiencia de cliente y hará que cada vez confiemos más en la tecnología. En referencia a esto, Gartner predice que aproximadamente el 30\% de la navegación web se realizará a través de la voz (y sin pantalla) para 2020, según un análisis de Dreamlt de abril de 2019 (Dreamit, 2019).

Los bancos encontrarán grandes ventajas en la Inteligencia Artificial en sus operaciones, ya que podrán mejorar la eficacia de sus modelos de gestión de riesgo de crédito como apunta Haycanal en su artículo "La Inteligencia Artificial en la gestión del riesgo de crédito"1 incorporando información de fuentes externas como por ejemplo datos de redes sociales o información histórica que les permitirá analizar patrones de comportamiento de otros clientes similares, o para planificar mejor sus flujos de caja y la gestión de proveedores. La Inteligencia Artificial también les permitirá prevenir el fraude detectando actividades sospechosas de movimiento de dinero o actividades inadecuadas de los clientes, y protegerse contra los ciberataques.

${ }^{1}$ https://haycanal.com/noticias/11698/lainteligencia-artificial-en-la-gestion-del-riesgo-de-credito) 
Adicionalmente, los bancos ya están utilizando la Inteligencia Artificial para aumentar la eficiencia de sus procesos y reducir costes operativos a la vez que mejoran la atención al cliente y la venta cruzada por cualquiera de sus canales. Esto lo llevan a cabo gracias a la automatización inteligente de procesos y al uso de soluciones cognitivas capaces de extraer, categorizar y analizar información para dar soporte a su operativa diaria, como por ejemplo recobro, soporte a operaciones transaccionales, embargos, cierres diarios, etc.

Gracias a la Inteligencia Artificial y a la gran cantidad de información que los bancos tienen hoy sobre sus clientes en un futuro los bancos serán capaces de ofrecer un servicio totalmente digital y personalizado porque contarán con el conocimiento casi total de sus clientes y con la tecnología necesaria para que realicen sus operaciones a través de cualquier dispositivo digital simplemente utilizando la voz, de tal modo que las sucursales físicas disminuirán en número o asumirán nuevas funciones que ahora ni imaginamos, por ejemplo, el banco Santander ha convertido una serie de sucursales en "work café" (Arroyo, 2019).

El sector asegurador está implementando el mismo tipo de soluciones digitales basadas en Inteligencia Artificial que la banca, aunque está invirtiendo mucho más en el desarrollo de modelos de precios de seguros personalizados, basados en nuevas modalidades de uso o en modelos que les dan la posibilidad de cubrir riesgos hasta ahora desconocidos.

Algunos ejemplos de estos nuevos modelos se están ensayando en México, en donde por ejemplo Mapfre² ya tiene un seguro de coche en el cual es el cliente el que elije las coberturas que más se adecuan a sus necesidades o $\mathrm{Miituo}^{3}$, que tiene un seguro de coche de pago por $\mathrm{Km}$, es decir es un pago por uso más beneficioso para el cliente y con menor riesgo para la aseguradora.

\section{CÓMO HACER QUE LOS NUEVOS SERVICIOS DE INNOVACION UTILICEN LA INTELIGENCIA ARTIFICIAL DE FORMA ÉTICA Y CONFIABLE}

En un futuro casi inmediato las empresas serán capaces de desarrollar cualquier aplicación basada en Inteligencia Artificial para mejorar y aumentar la eficiencia de su negocio, pero ¿cómo sabremos si lo estarán haciendo de forma ética? ¿Cómo sabremos que las decisiones que tome un sistema autónomo de Inteligencia Artificial serán decisiones correctas y sin sesgos?

Debemos ser conscientes de que un mal uso de la Inteligencia Artificial o la ausencia de principios éticos en su aplicación puede conllevar una serie de riesgos potenciales para las empresas como el riesgo reputacional y dañar o perjudicar a las personas por ejemplo con una scoring de riesgo negativo.

Por eso no debemos dejar de ver que la automatización de tareas, la digitalización, las aplicaciones de Inteligencia Artificial y el acceso a datos deben ser usadas siempre en favor y protegiendo los derechos de la persona a la que van dirigidas mediante la aplicación de una serie de medidas que eviten los sesgos por el uso de datos históricos en los algoritmos y que cumplan con una serie de principios que garanticen su buen uso.

\footnotetext{
${ }^{2}$ https://www.mapfre.com.mx/seguros-mx/particulares/seguros-de-auto/auto/tu-eliges/

${ }^{3}$ https://www.miituo.com/
} 
La Comisión Europea publicó el pasado 19 de febrero la Estrategia Digital Europea, basada en el valor de los datos, junto con el Libro Blanco de Inteligencia Artificial (Comisión Europea, 2020). Ambos documentos fueron definidos por la presidenta de la Comisión, Úrsula von der Leyen, como un marco regulador basado en valores fundamentales y capaz de convertir a Europa en líder mundial de la innovación en la economía de los datos y sus aplicaciones con una estrategia centrada en el ser humano. En palabras de la mandataria comunitaria, "Europa debería apostar por una tecnología que redunde en el beneficio de las personas, una economía justa y competitiva y una sociedad abierta, democrática y sostenible".

Está claro que la Inteligencia Artificial va a transformar nuestras vidas y que en este momento nos presenta muchas oportunidades, pero también muchas amenazas debido a un mal uso de la información o por la toma de decisiones sesgadas o que no puedan ser explicadas. Por eso, no podemos dejar que nuestra seguridad y la toma de decisiones sea manejada por los algoritmos, porque una misma aplicación de Inteligencia Artificial podría ser usada con fines malintencionados teniendo efectos imprevistos para las personas. Para ello, será necesario que el uso de la Inteligencia Artificial se regule según las directrices de la Comisión Europea que velen por un uso confiable para los ciudadanos y las empresas. 


\section{REFERENCIAS BIBLIOGRÁFICAS}

- Arroyo, R. (5 de diciembre de 2019). Expansión. Los bancos reinventan las sucursales. https://www. expansion.com/empresas/inmobiliario/2019/12/05/5de81aece5fdea44108b4672.html

- Comisión Europea. (2020). Libro Blanco sobre la inteligencia artificial: un enfoque europeo orientado a la excelencia y la confianza. https://ec.europa.eu/info/sites/info/files/commission-white-paper-artificialintelligence-feb2020_es.pdf

- Cornieles, P. (31 de julio de 2019). Ialatam. Inteligencia Artificial, robots y biotecnología, así es el futuro de la agricultura. https://ia-latam.com/2019/07/31/inteligencia-artificial-robots-y-biotecnologia-asi-es-elfuturo-de-la-agricultura/

- Dreamit. (23 de abril de 2019). The startups and investors bringing voice tech to healthcare. https://www. dreamit.com/journal/voice-tech-healthcare

- Haycanal. La Inteligencia Artificial en la gestión del riesgo de crédito. https://haycanal.com/noticias/11698/ la-inteligencia-artificial-en-la-gestion-del-riesgo-de-credito\#: :text=La\%2OIA\%20mejora\%20entre\%20 un,gesti\%C3\%B3n\%20del\%20riesgo\%20de\%20cr\%C3\%A9dito.\&text=Pero\%20la\%2OInteligencia\%20 Artificial\%20se,el\%20sector\%20financiero\%3A\%20la\%20ciberseguridad.

- Mapfre. Seguro de auto Tú eliges. https://www.mapfre.com.mx/seguros-mx/particulares/seguros-de-auto/ auto/tu-eliges/

- Miituo. https://www.miituo.com/

- Porras, J.(18 de febrero de 2020). Robo-Advisors. Inteligencia Artificial parafacilitar la inversión, blog Unicajabanco. https://uniblog.unicajabanco.es/robo-advisors--inteligencia-artificial-para-facilitar-la-inversi

- Roca JM. (julio de 2019). Multinacionales marca España. La digitalización y la IA en España: Una perspectiva desde el ámbito educativo. https://multinacionalesmarcaespana.org/wp-content/uploads/2019/07/ Informe_IA_EDUCACION.pdf

- SánchezM.(17 defebrerode 2020). El faradio. MétodoSancal, el pianoquetoca contrael envejecimiento neuronal. https://www.elfaradio.com/2020/02/17/metodo-sancal-el-piano-que-toca-contra-el-envejecimientoneuronal/\#: :text=El\%20M\%C3\%A9todo\%20S\%C3\%A1ncal\%20es\%20una,ejemplo\%2C\%20 disfrutan\%20tocando\%20el\%20piano.

- Trendov, N., Varas, S., \& Zeng, M. (2019). Tecnologías digitales en la agricultura y las zonas rurales. División de Tecnología de la Información. Organización de las Naciones Unidas para la Alimentación y la Agricultura (FAO), 1-26. http://www.fao.org/3/ca4887es/ca4887es.pdf 
REVISTA DIECISIETE
NOTAS Y COLABORACIONES 